\title{
A Sinusoidally Driven Lorenz System and Circuit Implementation
}

\author{
Chunyan Han, ${ }^{1,2}$ Simin Yu, ${ }^{1}$ and Guangyi Wang ${ }^{3}$ \\ ${ }^{1}$ School of Automation, Guangdong University of Technology, Guangzhou 510006, China \\ ${ }^{2}$ Department of Physics and Electronic Science, Binzhou University, Binzhou 256603, China \\ ${ }^{3}$ School of Electronics and Information, Hangzhou Dianzi University, Hangzhou 310018, China
}

Correspondence should be addressed to Chunyan Han; cyh660@163.com

Received 3 February 2015; Revised 6 May 2015; Accepted 10 May 2015

Academic Editor: Oded Gottlieb

Copyright (C) 2015 Chunyan Han et al. This is an open access article distributed under the Creative Commons Attribution License, which permits unrestricted use, distribution, and reproduction in any medium, provided the original work is properly cited.

\begin{abstract}
Another approach is developed for generating two-wing hyperchaotic attractor, four-wing chaotic attractor, and high periodic orbits such as period-14 from a sinusoidally driven based canonical Lorenz system. A sinusoidal function controller is introduced into a 3D autonomous Lorenz system, so that the abovementioned various hyperchaotic attractors, chaotic attractors, and high periodic orbits can be obtained, respectively, by adjusting the frequency of the sine function. In addition, an analog circuit and a digital circuit are also designed and implemented, with experimental results demonstrated. Both numerical simulations and circuit implementation together show the effectiveness of the proposed systematic methodology.
\end{abstract}

\section{Introduction}

In recent years, chaos has been widely studied in various fields, including mathematics, physics, biology, and engineering sciences. In retrospect, in 1963, Lorenz found the first numerical chaotic system with a clear physical meaning [1]. In 1979, Rössler found the first chaotic system with two positive Lyapunov exponents, referred to as the Rössler hyperchaotic system [2]. Comparing with a chaotic attractor with one positive Lyapunov exponent, the hyperchaotic attractor expands in two or more directions simultaneously [3-5]. It means that the hyperchaotic attractor has much more complex topological structure and therefore has much better performances in many real-world applications such as secure communication and encryption. Moreover, synchronization of the fractional order hyperchaos Lorenz systems with activation feedback control, projective synchronization of fractional order chaotic system based on linear separation, observer-based decentralized fuzzy neural sliding mode control for interconnected unknown chaotic systems via network structure adaptation, and so on have been deeply investigated [6-8]. These new chaotic systems are superior in encryption and can enhance security for multimedia chaotic secure communications.

Based on the 3D autonomous Lorenz system, considerable efforts have been devoted to constructing various hyperchaotic Lorenz systems with two positive Lyapunov exponents [9-17]. The related proposed methods mainly involve two aspects: one is with respect to the $4 \mathrm{D}$ autonomous Lorenz system based on state feedback control approach [9-13] and another is related to the 3D nonautonomousbased Lorenz system with parameters perturbation or control term by using continuously differentiable periodic functions, such as sine and cosine functions [14-16]. In addition, a $4 \mathrm{D}$ nonautonomous-based Lorenz system is also proposed for generating hyperchaos with two positive Lyapunov exponents [17]. Using a sinusoidally driven chaotic system to generate hyperchaos is a simple effective approach. Recently, it is shown that when a sinusoidal voltage stimulus is applied to a memristor-based Chua's circuit, the memristive Chua's circuit can exhibit hyperchaotic attractor [18].

Moving forward from the abovementioned accomplished works and taking into account the actual circuit implementation, in this paper, another approach is further developed 
for generating two-wing hyperchaotic attractor, four-wing chaotic attractor, and high periodic orbits such as period14 , from a sinusoidally driven Lorenz system. More precisely, a sinusoidal function controller is introduced into the first equation of 3D autonomous Lorenz system, which is different from the proposed parameters perturbation or control term [14-16], or 4D nonautonomous-based approach [17]. Comparing the abovementioned existing reports with our proposed method, the main differences lie in the following two different ways. (i) Our proposed controlled system can be considered and constructed not only for generating twowing hyperchaotic attractor, which is also reported in the existing literatures [14-17], but also for four-wing chaotic attractor and high periodic orbits such as period-14, through adjusting the frequency of the sine function. (ii) From the viewpoint of circuit implementation, the accuracy of frequency adjustment is usually higher than that of parameter control, and it is more convenient for circuit parameter adjustment after considering the variable proportion compression transformation and time scaling. Because of this, the abovementioned various hyperchaotic attractors, chaotic attractors, and high periodic orbits can be easily observed from designed circuit experiment. As a comparison, for 4D autonomous-based state feedback control approach [913], circuit experimental results have shown that it is more difficult for generating high periodic orbits such as period14 from circuit implementation, since it belongs to parameter control instead of frequency adjustment. Furthermore, a module-based unified circuit diagram is also designed for implementing the abovementioned various hyperchaotic attractors, chaotic attractors, and periodic orbits as several typical examples for illustration and demonstration.

The rest of the paper is organized as follows. Design of a sinusoidally driven Lorenz system is introduced in Section 2. The basic dynamic behaviors are analyzed in Section 3. A module-based unified circuit diagram is designed for implementing various hyperchaotic attractors, chaotic attractors, and periodic orbits in Section 4. Finally, Section 5 concludes the paper.

\section{Design of a Sinusoidally Driven Lorenz System}

Lorenz system is described as follows:

$$
\begin{aligned}
& \dot{x}=-A(x-y), \\
& \dot{y}=B x-x z-y \\
& \dot{z}=x y-D z,
\end{aligned}
$$

where $A, B$, and $D$ are parameters of Lorenz system. By introducing a sinusoidal function controller into the first equation of system (1), one gets a sinusoidally driven Lorenz system, given by

$$
\begin{aligned}
& \dot{x}=-A(x-y-a \sin \omega t), \\
& \dot{y}=B x-x z-y, \\
& \dot{z}=x y-D z,
\end{aligned}
$$

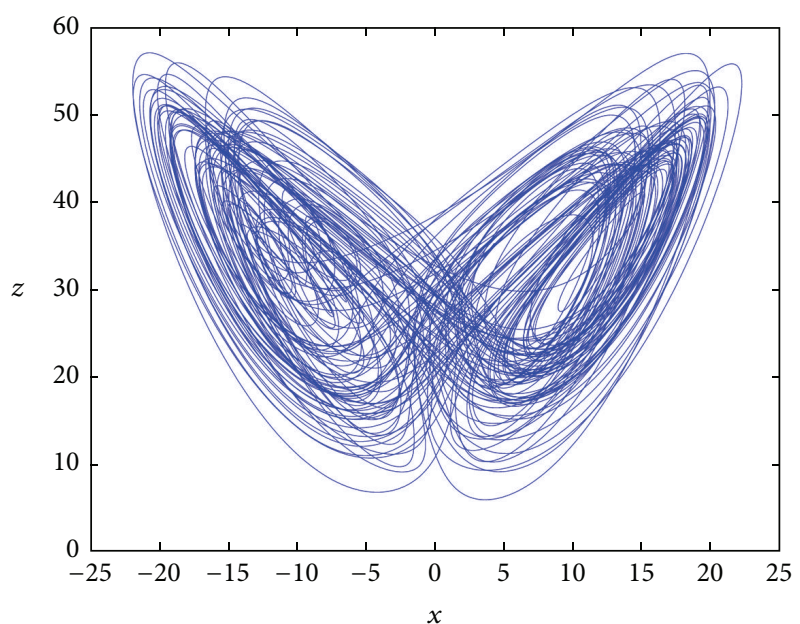

FIGURE 1: The two-wing hyperchaotic attractor.

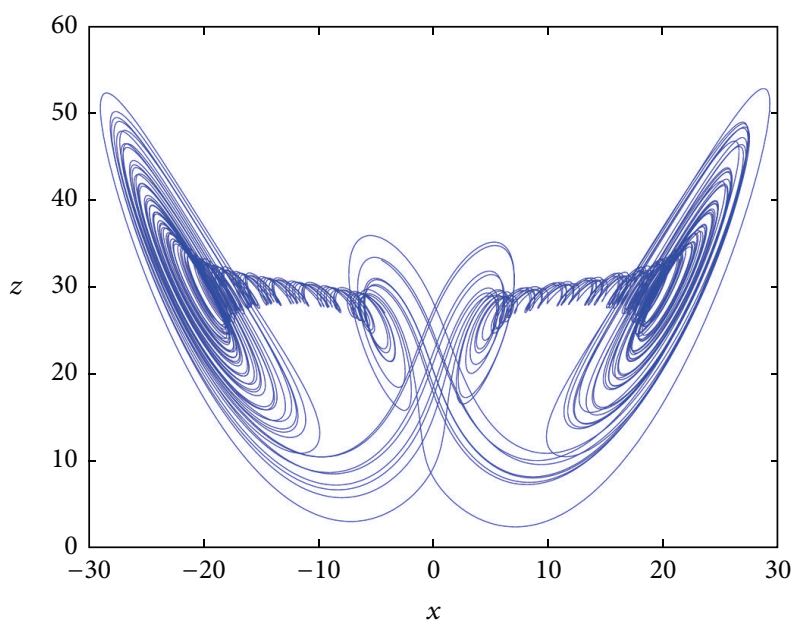

FIgURE 2: The four-wing chaotic attractor with $A=10, B=30$, $D=8 / 3, a=17$, and $\omega=0.5$.

where $a \sin \omega t$ is the sinusoidal function controller and $a$ and $\omega$ are the amplitude and frequency of the sine function controller, respectively.

According to (2) and letting $u=\omega t$, the corresponding $4 \mathrm{D}$ autonomous Lorenz system is given by

$$
\begin{aligned}
& \dot{x}=-A(x-y-a \sin u), \\
& \dot{y}=B x-x z-y, \\
& \dot{z}=x y-D z, \\
& \dot{u}=\omega .
\end{aligned}
$$

(i) Let $A=8, B=35, D=8 / 3, a=6$, and $\omega=$ 4.5. System (3) generates a two-wing hyperchaotic attractor, as shown in Figure 1.

(ii) Let $A=10, B=30, D=8 / 3, a=17$, and $\omega=0.5$. System (3) generates a four-wing chaotic attractor, as shown in Figure 2. 
(iii) Let $A=10, B=30, D=8 / 3, a=17$, and $\omega=$ $1.03,1.47,2.5,3.8,7.6$. System (3) generates period-14, period-10, period- 6 , period-4, and period- 2 orbits, respectively, as shown in Figure 3.

\section{Analysis of Basic Dynamical Behaviors}

In this subsection, the basic dynamic behaviors of the proposed sinusoidally driven Lorenz system are analyzed, including equilibrium points with time evolution, Lyapunov exponents, and bifurcation with the frequency variation of the sine function controller.

3.1. Equilibrium Points with Time Evolution. According to system (2), the equilibrium point equation is given by

$$
\begin{aligned}
x_{e}^{3}+b x_{e}^{2}+c x_{e}+d & =0, \\
y_{e} & =x_{e}+b, \\
z_{e} & =\frac{\left(x_{e}^{2}+b x_{e}\right)}{D},
\end{aligned}
$$

where $b=-a \sin \omega t, c=D(1-B)$, and $d=-D a \sin \omega t$.

Letting $x_{e}=X_{e}-b / 3$ and substituting it into the first equation of (4), one gets

$$
X_{e}^{3}+p X_{e}+q=0
$$

where $p=c-b^{2} / 3$ and $q=2 b^{3} / 27-c b / 3+d$.

According to Cardan discriminant $\Delta=(q / 2)^{2}+(p / 3)^{3}$, when $\Delta>0$, there exist a real root and two complex roots in (5). Since the equilibrium points cannot be complex numbers, this means that the system does not have equilibrium points since $\Delta>0$.

Similarly, when $\Delta \leq 0$, there exist three real roots in (5), and this means that the system has three equilibrium points since $\Delta \leq 0$, given by

$$
\begin{aligned}
& x_{1 e}=\mu_{2} \times \sqrt[3]{-\frac{q}{2}+\sqrt{\Delta}}+\mu_{1} \times \sqrt[3]{-\frac{q}{2}-\sqrt{\Delta}}-\frac{b}{3}, \\
& y_{1 e}=x_{1 e}+b \\
& z_{1 e}=\frac{\left(x_{1 e}^{2}+b x_{1 e}\right)}{D}, \\
& x_{2 e}=\sqrt[3]{-\frac{q}{2}+\sqrt{\Delta}}+\sqrt[3]{-\frac{q}{2}-\sqrt{\Delta}}-\frac{b}{3}, \\
& y_{2 e}=x_{2 e}+b \\
& z_{2 e}=\frac{\left(x_{2 e}^{2}+b x_{2 e}\right)}{D}, \\
& x_{3 e}=\mu_{1} \times \sqrt[3]{-\frac{q}{2}+\sqrt{\Delta}}+\mu_{2} \times \sqrt[3]{-\frac{q}{2}-\sqrt{\Delta}}-\frac{b}{3}, \\
& y_{3 e}=x_{3 e}+b, \\
& z_{3 e}=\frac{\left(x_{3 e}^{2}+b x_{3 e}\right)}{D},
\end{aligned}
$$

where $\mu_{1}=(-1+j \sqrt{3}) / 2$ and $\mu_{1}=(-1-j \sqrt{3}) / 2$.
For two-wing hyperchaotic attractor, let $A=8, B=35$, $D=8 / 3, a=6$, and $\omega=4.5$. According to Cardan's discriminant $\Delta=(q / 2)^{2}+(p / 3)^{3}$, the simulation result of $\Delta$ with time evolution is shown in Figure 4 . One can see that $\Delta$ is a periodic function with time, and $\Delta<0$ is always satisfied. According to (5)-(6), there exist three trajectories of saddle focus equilibrium points with time evolution, as shown in Figure 5, where the centers (denoted by two red hollow circles) of two wings are located on the trajectories.

Similarly, as for four-wing chaotic attractor, let $A=10$, $B=30, D=8 / 3, a=17$, and $\omega=0.5$. According to Cardan's discriminant $\Delta=(q / 2)^{2}+(p / 3)^{3}$, one gets the simulation result of $\Delta$ with time evolution, as shown in Figure 6. One can see that $\Delta$ is also a periodic function with time, and $\Delta<0$ is always satisfied. According to (5)-(6), there exist three trajectories of saddle focus equilibrium points with time evolution, as shown in Figure 7. The centers (denoted by four red hollow circles) of four wings are located on the trajectories, alternately switching among these four equilibrium points with time evolution, so that a four-wing chaotic attractor can be created.

3.2. Lyapunov Dimension. When parameters $A=8, B=35$, $D=8 / 3, a=6$, and $\omega=4.5$, the corresponding Lyapunov exponents of this hyperchaotic attractor are

$$
\begin{aligned}
& \mathrm{LE}_{1}=0.8058, \\
& \mathrm{LE}_{2}=0.1216, \\
& \mathrm{LE}_{3} \approx 0, \\
& \mathrm{LE}_{4}=-12.5986 .
\end{aligned}
$$

The Lyapunov dimension of an attractor is defined by its Lyapunov exponents; that is,

$$
D_{L}=j+\frac{\sum_{i=1}^{j} \mathrm{LE}_{i}}{\left|\mathrm{LE}_{j+1}\right|},
$$

where $j$ is an integer which satisfies $\sum_{i=1}^{j} \mathrm{LE}_{i} \geq 0$ and $\sum_{i=1}^{j+1} \mathrm{LE}_{i}<0$.

With the Lyapunov dimension of the above hyperchaotic attractor being $D_{L}=3.0740$, this means that the hyperchaotic attractor has fractal dimension.

\subsection{Lyapunov Exponent and Bifurcation versus Frequency and} Amplitude. Let $A=8, B=35, D=8 / 3$, and $a=6$. According to system (3), the Lyapunov exponent spectrum LE versus frequency $\omega$ is obtained, as shown in Figure 8. When $1.1<\omega<5.9, \mathrm{LE}_{1}>0, \mathrm{LE}_{2}>0, \mathrm{LE}_{3}=0$, and $\mathrm{LE}_{4}<0$, system (3) is hyperchaotic while in the parameter intervals $0<\omega<1.1$ and $5.9<\omega<10$ this system maintains chaotic characteristics of Lorenz system. By choosing $\omega=4.5$, a two-wing hyperchaotic attractor is generated, as shown in Figure 1.

When $A=8, B=35, D=8 / 3$, and $\omega=4.5$, the Lyapunov exponent spectrum with respect to amplitude $a$ is shown in 


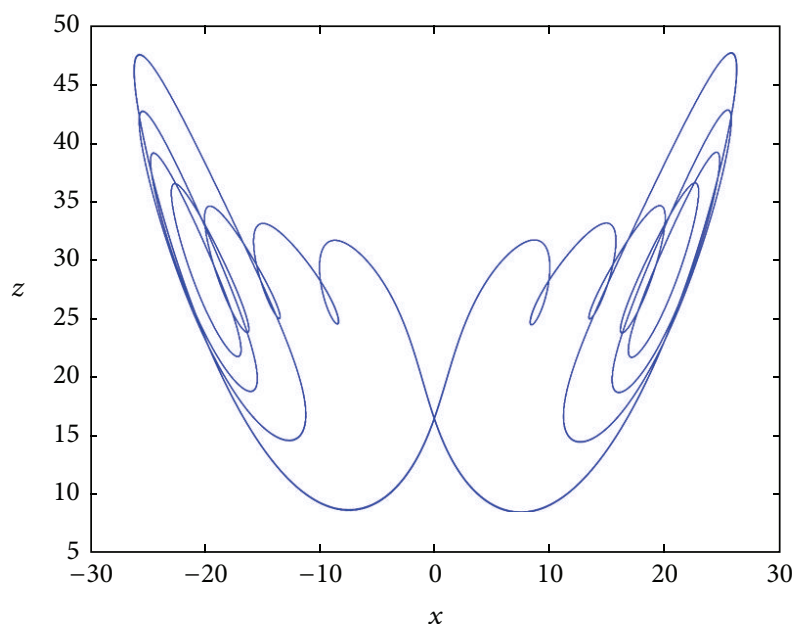

(a) Period-14 orbit with $\omega=1.03$

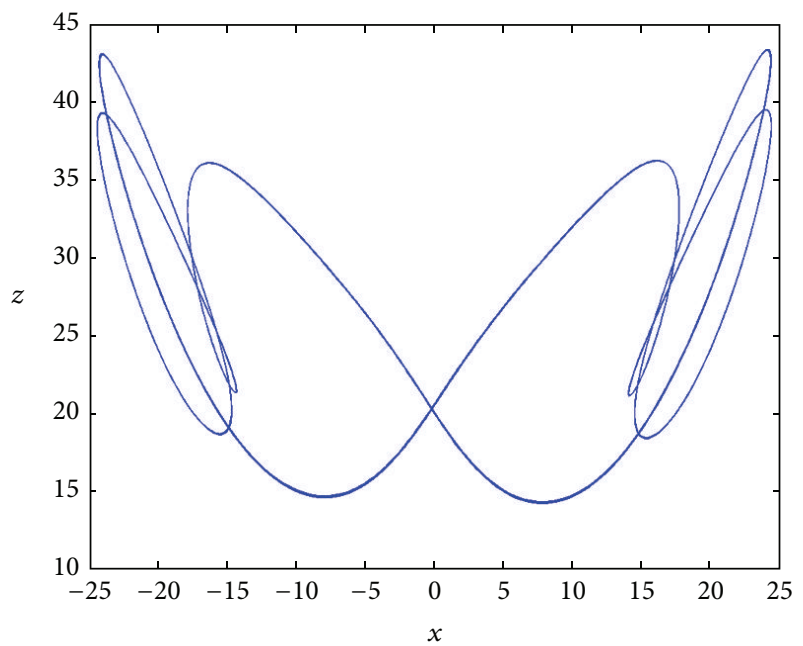

(c) Period-6 orbit with $\omega=2.5$

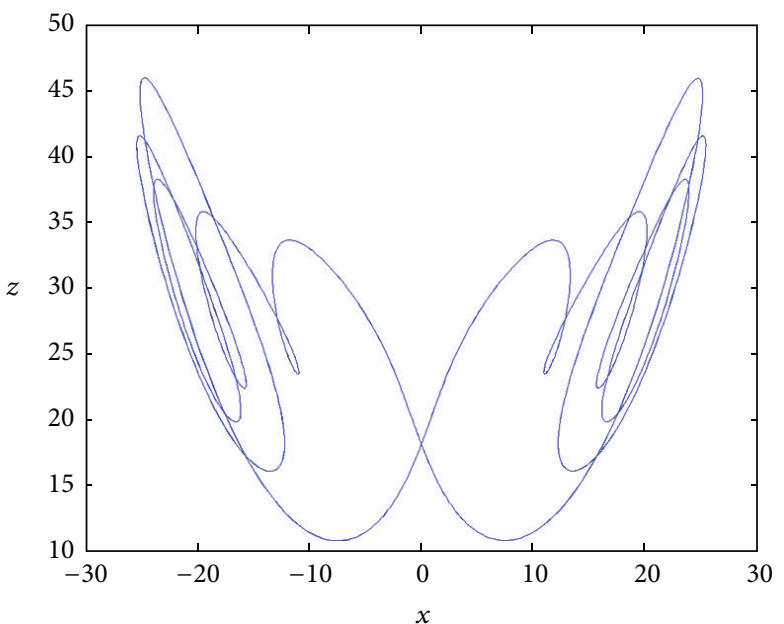

(b) Period-10 orbit with $\omega=1.47$

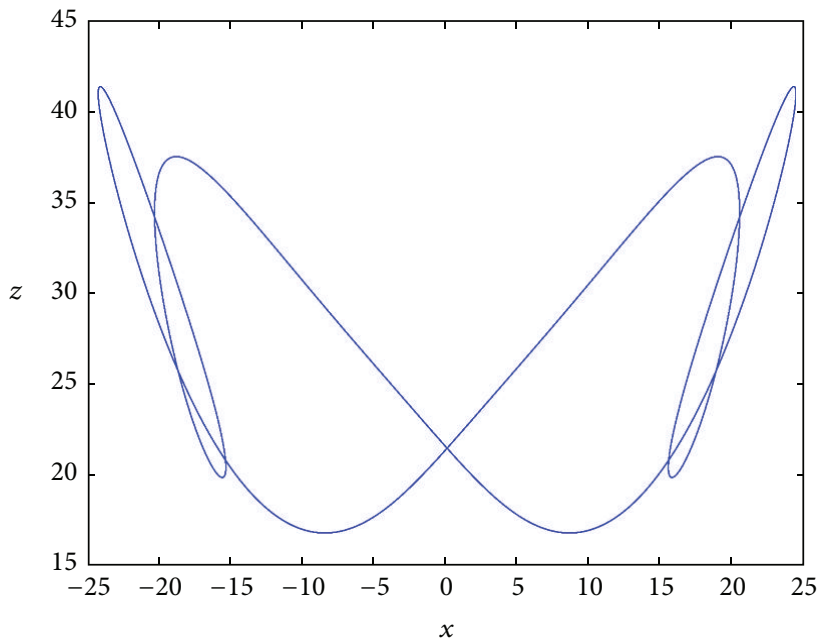

(d) Period-4 orbit with $\omega=3.8$

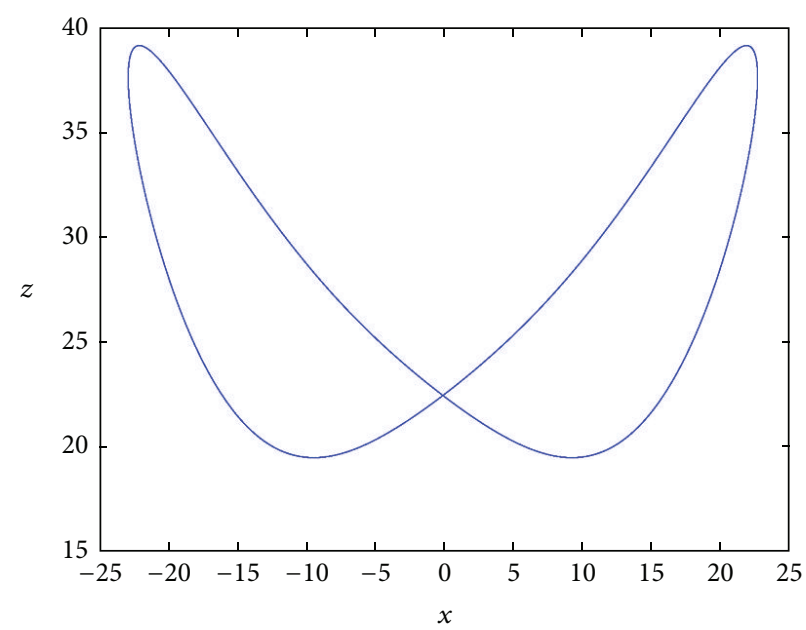

(e) Period-2 orbit with $\omega=7.6$

FIGURE 3: The period-14, period-10, period-6, period-4, and period-2 orbits. 


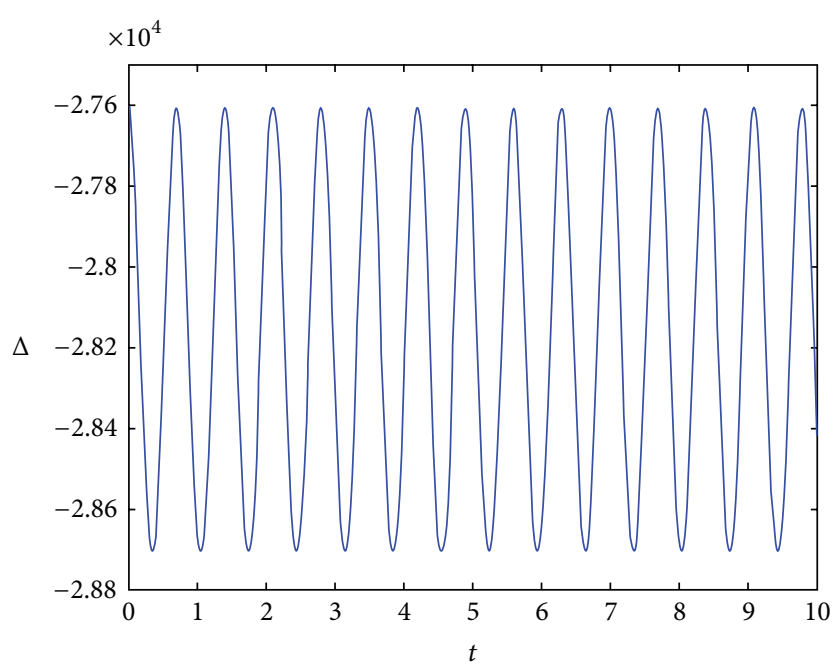

FIgURE 4: $\Delta<0$ with time evolution for two-wing hyperchaotic attractor.

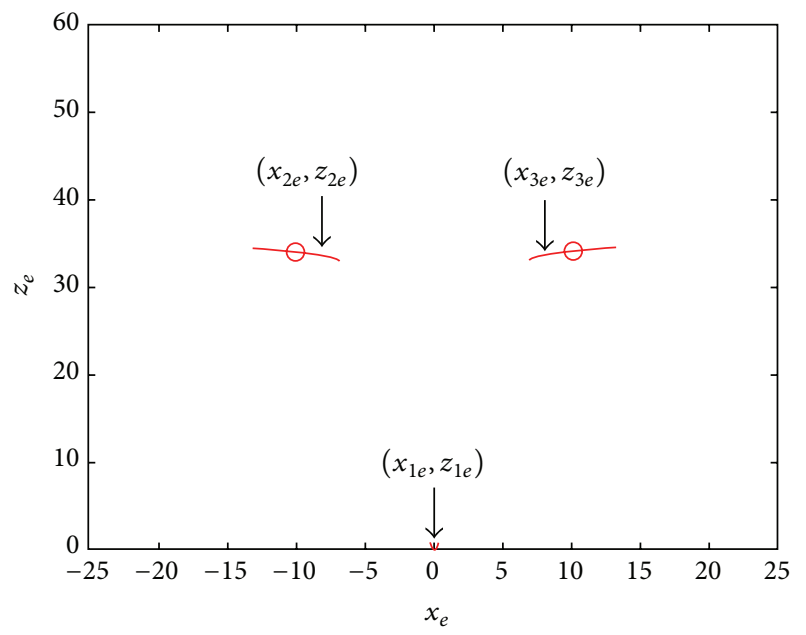

(a) Trajectories of equilibrium points

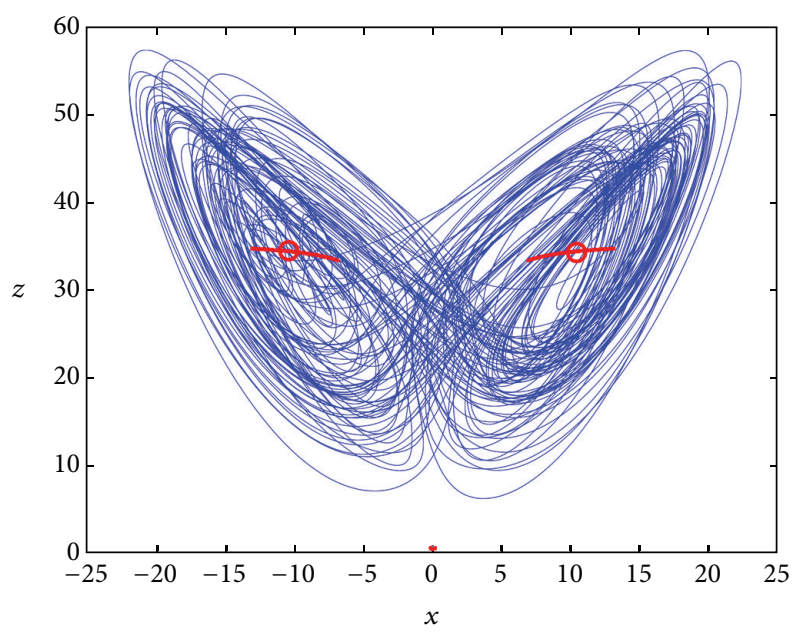

(b) Equilibrium points of two-wing hyperchaotic attractor

FIGURE 5: Three trajectories of equilibrium point corresponding to two-wing hyperchaotic attractor with time evolution.

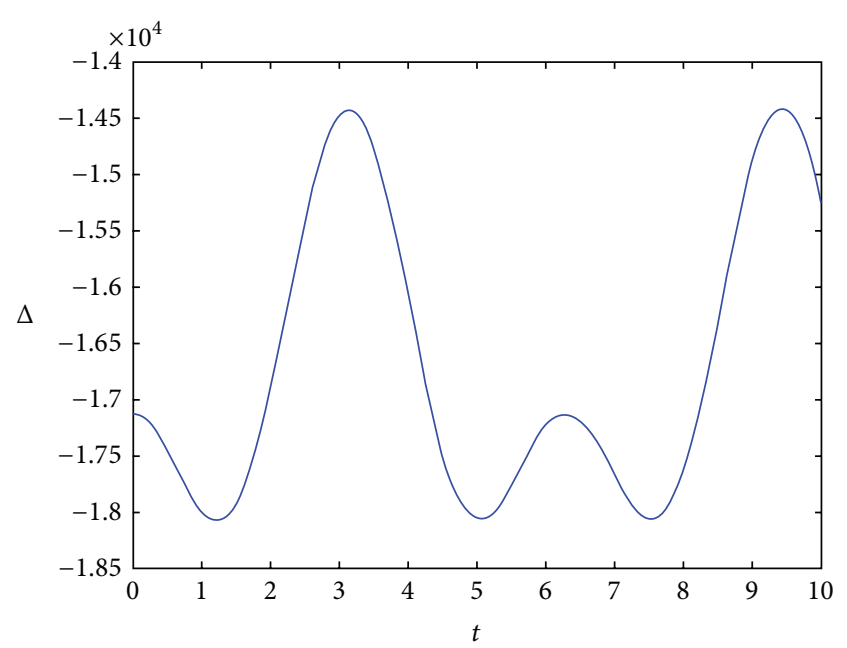

FIGURE 6: $\Delta<0$ with time evolution for four-wing chaotic attractor.

Figure 9, from which we can see that this system has also a hyperchaotic attractor in the parameter interval $1.11<a<$ 8.81.

Now we give the dynamical map of system (3) for simultaneously observing the bifurcation of $x$ with frequency $\omega$ and amplitude $a$ as shown in Figure 10, in which the blue region with the label $\mathrm{h}$ denotes hyperchaotic state, while the yellow region with the label $c$ denotes the chaotic state and the red one denotes the periodic state. From Figure 10 we can see that this system exhibits hyperchaotic behaviour in a large region of parameters $\omega$ and $a$, and it is good for generating more complex chaotic signals.

Let $A=10, B=30, D=8 / 3$, and $a=17$. According to system (3), the Lyapunov exponent spectrum LE and bifurcation versus frequency $\omega$ are drawn, as shown in Figures 11 (a) and 11(b), respectively. When $\omega=0.5, \mathrm{LE}_{1}>0, \mathrm{LE}_{2}<0$, $\mathrm{LE}_{3}=0$, and $\mathrm{LE}_{4}<0$, system (3) is chaotic, and a fourwing chaotic attractor is generated, as shown in Figure 2. It is noticed that there exist many periodic windows in the chaotic region, switching between chaotic state and periodic orbit with the increasing of frequency $\omega$. For example, by choosing $\omega=1.03,1.47,2.5,3.8,7.6$, system (3) generates period-14, period-10, period-6, period-4, and period-2 orbits, respectively, as shown in Figure 3.

To observe the periodic behaviors, the periodic window is expanded in the parameter interval $2.5<\omega<4$, as shown in Figure 12. Obviously, there are three kinds of bifurcation in the periodic window, including a perioddoubling bifurcation when $\omega \cong 3.45$, a pitchfork bifurcation when $\omega \cong 3.68$, and a tangent bifurcation when $\omega \cong 3.74$.

\section{Circuit Design and Implementation}

In this subsection, a module-based circuit design approach is proposed for realizing two-wing hyperchaotic attractor, 


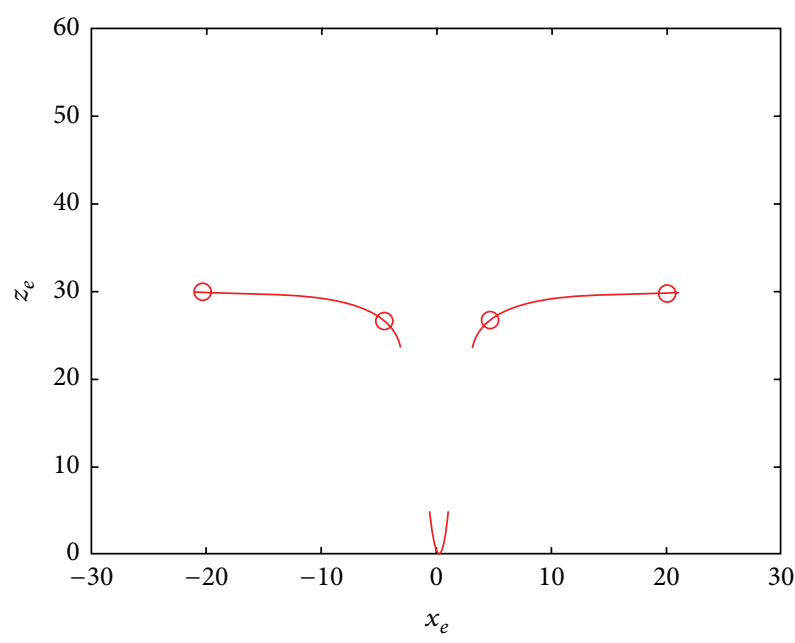

(a) Trajectories of equilibrium points

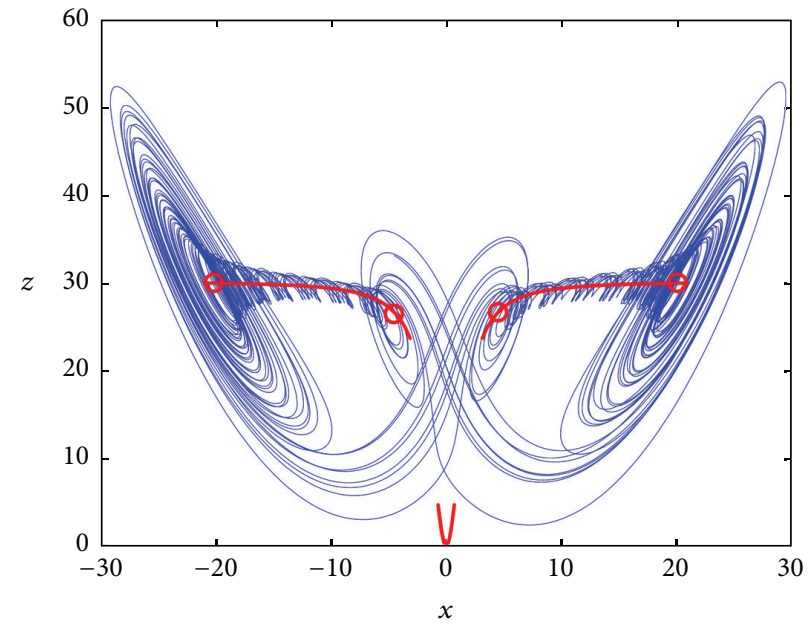

(b) Equilibrium points of four-wing chaotic attractor

FIGURE 7: Three trajectories of equilibrium point corresponding to four-wing chaotic attractor with time evolution.
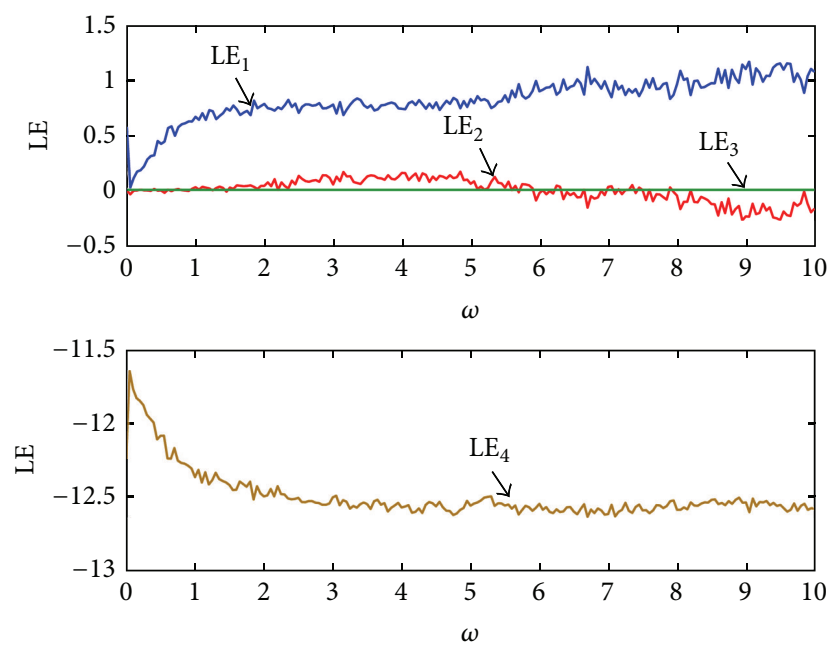

Figure 8: The diagram of LE versus $\omega(A=8, B=35, D=8 / 3$, and $a=6$ ).

four-wing chaotic attractor, and period-14, period-10, period-6, period-4, and period-2 orbits, respectively, and the corresponding experimental observations are also given.

4.1. Analog Circuit Design and Implementation. According to system (2), a circuit diagram for sinusoidally driven Lorenz system is designed, as shown in Figure 13. The circuit is mainly composed of integrator, adder, multiplier, inverter, and function signal generator.
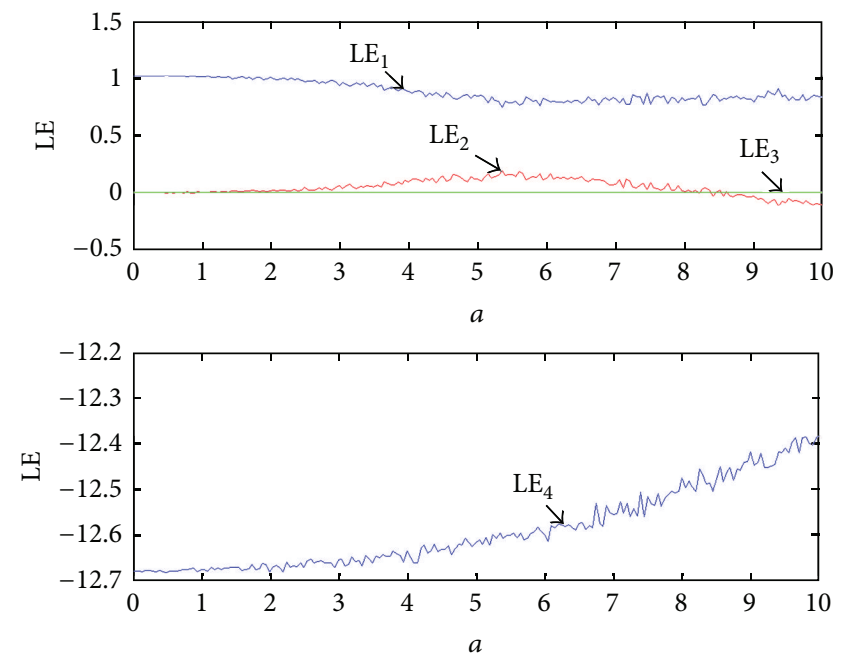

FIgURE 9: The diagram of LE versus $a(A=8, B=35, D=8 / 3$, and $\omega=4.5$ ).

According to Figure 13, one gets the state equations as follows:

$$
\begin{aligned}
& \frac{d x}{d \tau}=\frac{1}{R C}\left(-\frac{R}{R_{1}} x+\frac{R}{R_{2}} y+\frac{R}{R_{3}} a \sin (\Omega t)\right), \\
& \frac{d y}{d \tau}=\frac{1}{R C}\left(\frac{R}{R_{4}} x-\frac{R}{10 R_{5}} x z-\frac{R}{R_{6}} y\right), \\
& \frac{d z}{d \tau}=\frac{1}{R C}\left(\frac{R}{10 R_{7}} x y-\frac{R}{R_{8}} z\right) .
\end{aligned}
$$




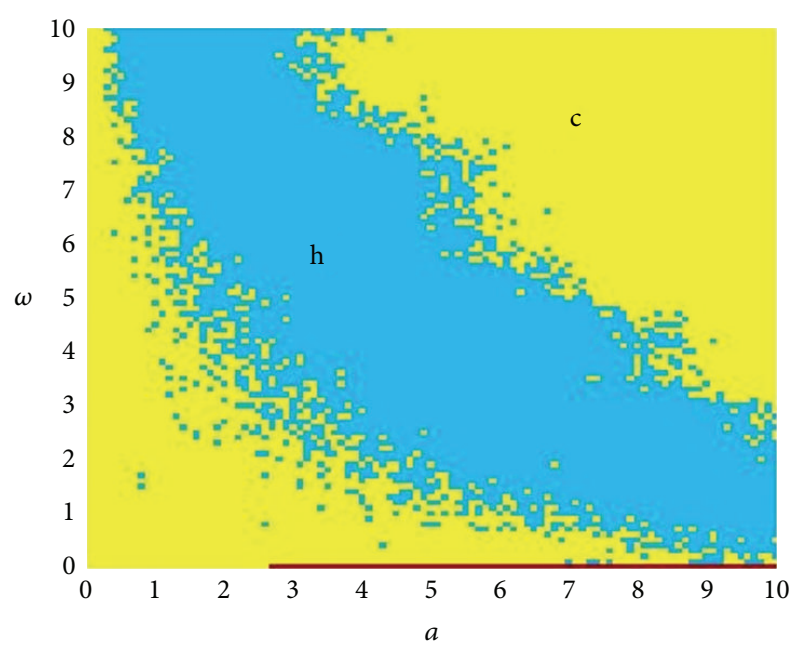

Figure 10: Dynamical map of system (3).
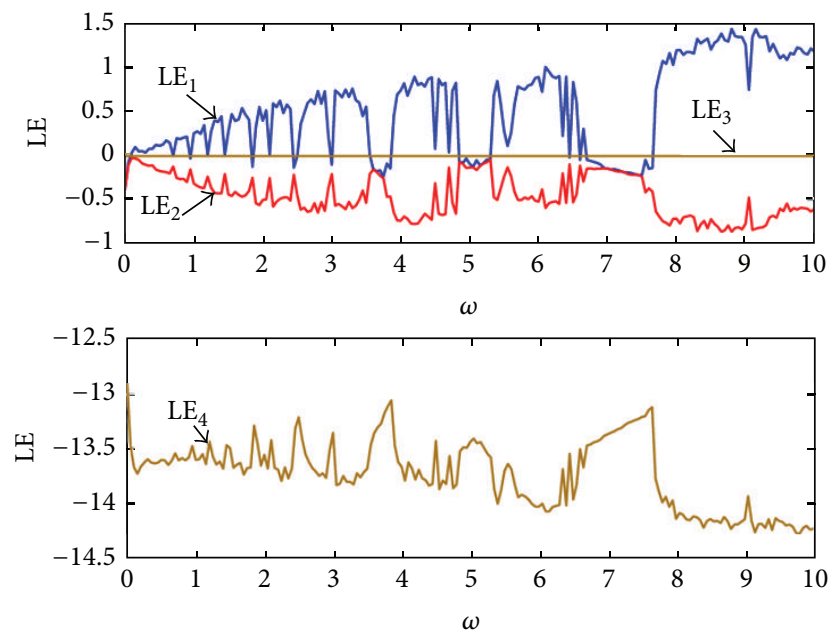

(a) The diagram of LE

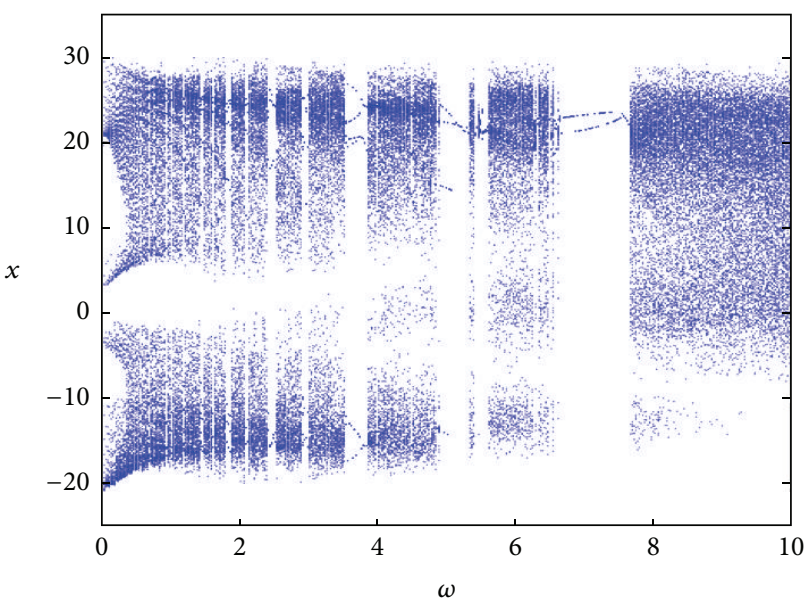

(b) The diagram of bifurcation

FIgURE 11: The diagram of LE and bifurcation versus $\omega(A=10$, $B=30, D=8 / 3$, and $a=17$ ).

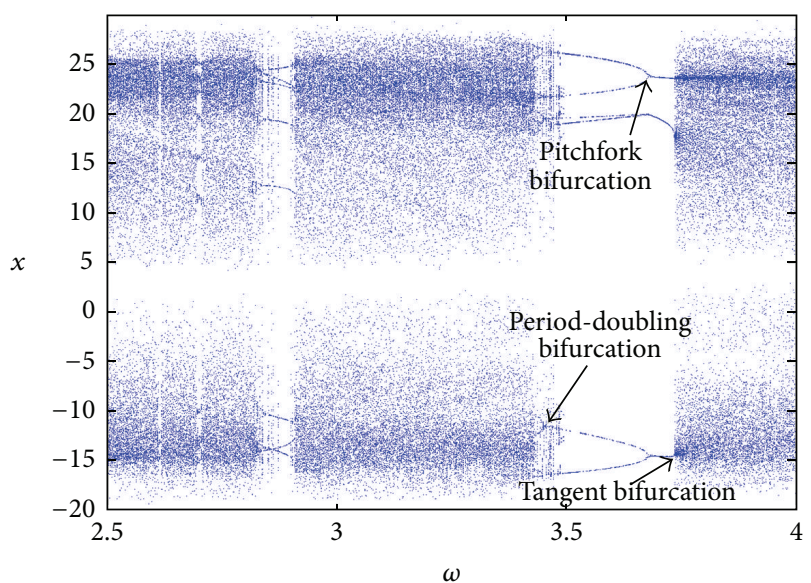

FIGURE 12: The diagram of bifurcation versus $\omega(A=10, B=30$, $D=8 / 3$, and $a=17$ ).

Doing the variable proportion compression transformation $x \rightarrow 0.1 x, y \rightarrow 0.1 y$, and $z \rightarrow 0.1 z$ and the time scale transformation $\tau=10 R C t$ on (9), respectively, one gets

$$
\begin{aligned}
& \frac{d(0.1 x)}{10 R C d t} \\
& =\frac{1}{R C}\left(-\frac{R}{R_{1}} 0.1 x+\frac{R}{R_{2}} 0.1 y+\frac{R}{R_{3}} a \sin (10 R C \Omega t)\right), \\
& \frac{d(0.1 y)}{10 R C d t}=\frac{1}{R C}\left(\frac{R}{R_{4}} 0.1 x-\frac{R}{10 R_{5}} 0.1^{2} x z-\frac{R}{R_{6}} 0.1 y\right), \\
& \frac{d(0.1 z)}{10 R C d t}=\frac{1}{R C}\left(\frac{R}{10 R_{7}} 0.1^{2} x y-\frac{R}{R_{8}} 0.1 z\right) .
\end{aligned}
$$

Let $R_{1}=R_{2}$. Therefore, (10) can be simplified, given by

$$
\begin{aligned}
& \frac{d x}{d t}=10 \frac{R}{R_{1}}(y-x)+100 \frac{R}{R_{3}} a \sin (10 R C \Omega t), \\
& \frac{d y}{d t}=\frac{10 R}{R_{4}} x-\frac{R}{10 R_{5}} x z-\frac{10 R}{R_{6}} y, \\
& \frac{d z}{d t}=\frac{R}{10 R_{7}} x y-\frac{10 R}{R_{8}} z .
\end{aligned}
$$

Comparing (11) with (2), one gets $A=10 R / R_{1}=100 R / R_{3}$, $B=10 R / R_{4}, D=10 R / R_{8}, 1=R /\left(10 R_{5}\right), 1=10 R / R_{6}$, $1=R /\left(10 R_{7}\right)$, and $\omega=10 R C \Omega$. Then all parameters can be determined as follows: $R_{1}=R_{2}=10 R / A, R_{3}=100 R / A$, $R_{4}=10 R / B, R_{8}=10 R / D, R_{5}=R_{7}=R / 10, R_{6}=10 R$, and $\Omega=\omega /(10 R C)$.

Choose $R=10 \mathrm{k} \Omega$ and $C=4.7 \mathrm{nF}$. Then one obtains $R_{1}=$ $R_{2}=100 / A(\mathrm{k} \Omega), R_{3}=1000 / A(\mathrm{k} \Omega), R_{4}=100 / B(\mathrm{k} \Omega), R_{8}=$ $100 / D(\mathrm{k} \Omega), R_{5}=R_{7}=1 \mathrm{k} \Omega, R_{6}=100 \mathrm{k} \Omega, \Omega=\omega /(10 R C)=$ $2.13 \times 10^{3} \omega$, and $f=\Omega /(2 \pi)=2.13 \times 10^{3} \omega /(2 \pi)$. 

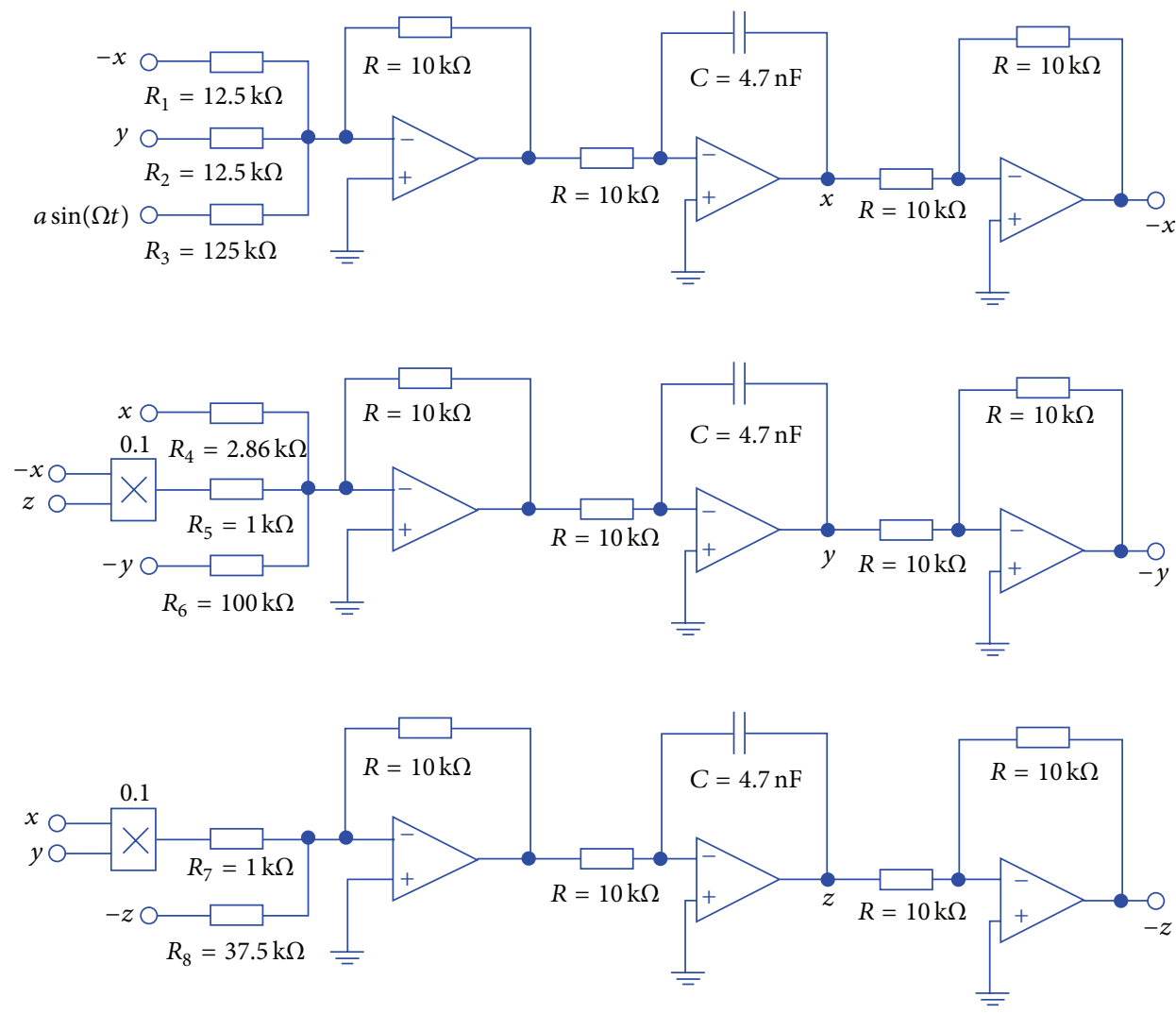

FIGURE 13: A module-based circuit diagram design according to (2).

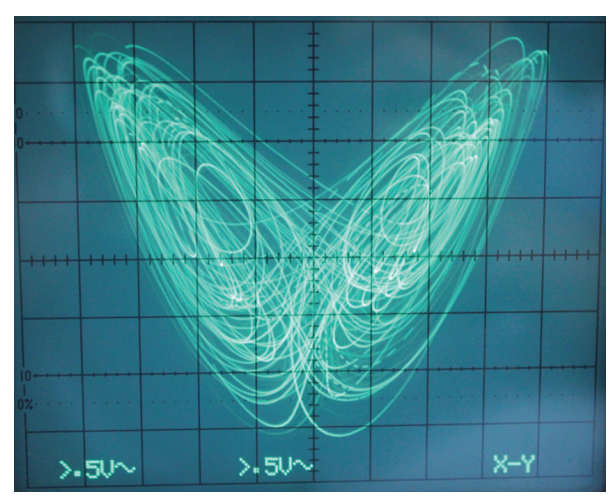

FIGURE 14: Circuit implementation result of two-wing hyperchaotic attractor.

(i) Let $A=8, B=35, D=8 / 3, a=6$, and $\omega=4.5$. Then one has $R_{1}=R_{2}=12.5 \mathrm{k} \Omega, R_{3}=125 \mathrm{k} \Omega$, $R_{4}=2.86 \mathrm{k} \Omega, R_{8}=37.5 \mathrm{k} \Omega, R_{5}=R_{7}=1 \mathrm{k} \Omega$, $R_{6}=100 \mathrm{k} \Omega$, and $f=1.53 \mathrm{kHz}$. According to Figure 13, the circuit implementation result of twowing hyperchaotic attractor is shown in Figure 14.

(ii) Let $A=10, B=30, D=8 / 3$, and $a=17$. Then one has $R_{1}=R_{2}=10 \mathrm{k} \Omega, R_{3}=100 \mathrm{k} \Omega, R_{4}=3.33 \mathrm{k} \Omega$,

$$
\begin{aligned}
& \begin{array}{l}
R_{5}=R_{7}=1 \mathrm{k} \Omega, R_{8}=37.5 \mathrm{k} \Omega, \text { and } R_{6}=100 \mathrm{k} \Omega . \\
\text { Denote }
\end{array} \\
& f=\frac{\Omega}{2 \pi}=\frac{2.13 \times 10^{3} \omega}{2 \pi}=\left\{\begin{array}{l}
169.5 \mathrm{~Hz}(\omega=0.5) \\
349.17 \mathrm{~Hz}(\omega=1.03) \\
498.33 \mathrm{~Hz}(\omega=1.47) \\
847.5 \mathrm{~Hz}(\omega=2.5) \\
1288.2 \mathrm{~Hz}(\omega=3.8) \\
2576.39 \mathrm{~Hz}(\omega=7.6) .
\end{array}\right.
\end{aligned}
$$

According to Figure 13, the circuit implementation results of four-wing chaotic attractor and period-14, period-10, period6 , period-4, and period-2 orbits are shown in Figures 15(a)15(f), respectively.

From Figures 8 and 9, obviously, the proposed system can generate the hyperchaotic attractor by adjusting the frequency $\omega$ of the sine function when $A=8, B=35$, $D=8 / 3$, and $a=6$ or by adjusting the amplitude $a$ of the sine function when $A=8, B=35, D=8 / 3$, and $\omega=4.5$. Simultaneously, from Section 4.1, one can see that the relation of the sinusoidal signal frequency $f$ in the circuit and the frequency $\omega$ in system (2) is $f=2.13 \times 10^{3} \omega /(2 \pi)$ after the variable proportion compression transformation and time scaling. It 


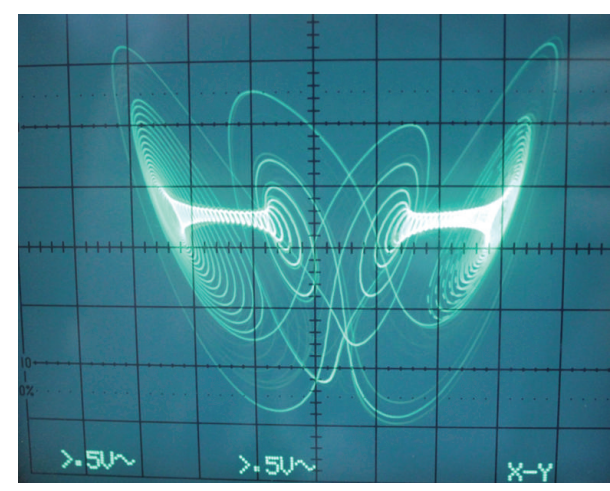

(a) Four-wing chaotic attractor

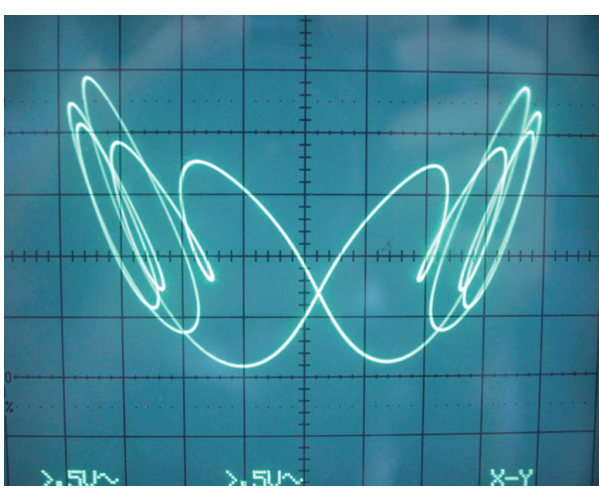

(c) Period-10 orbit

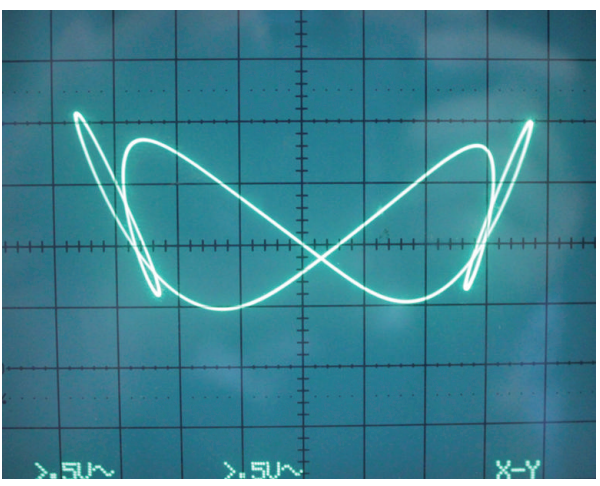

(e) Period-4 orbit

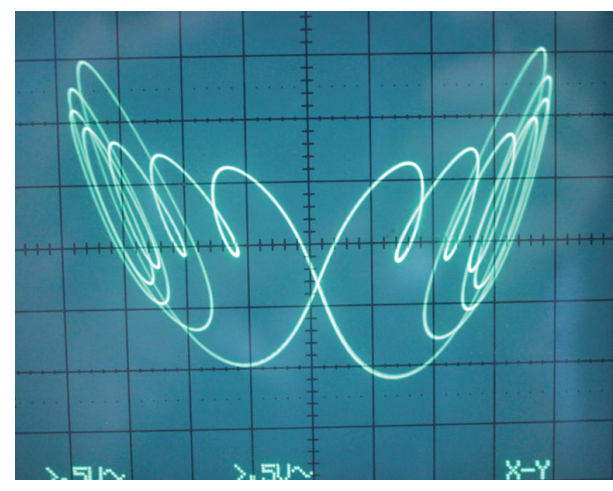

(b) Period-14 orbit

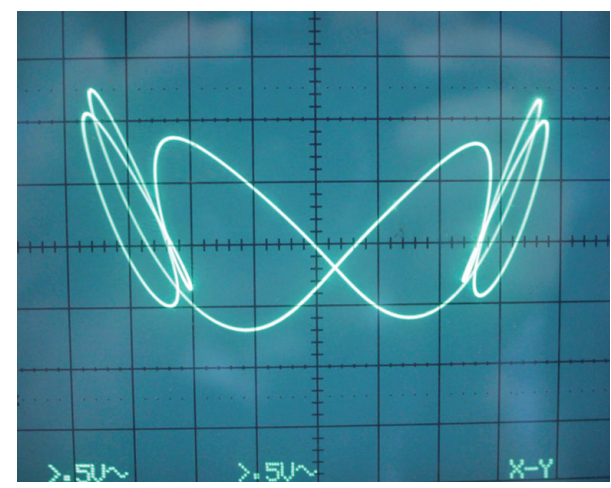

(d) Period-6 orbit

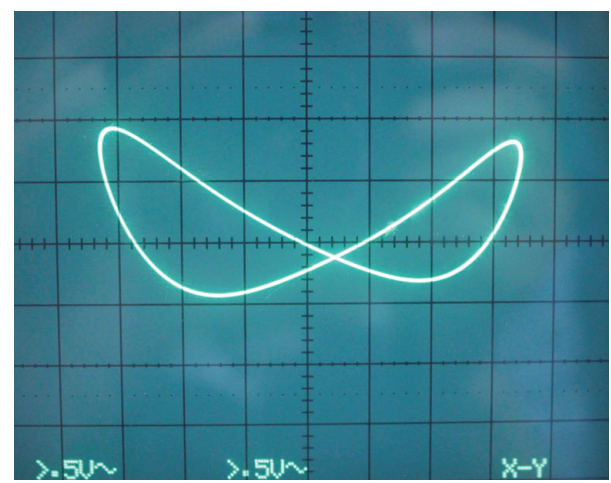

(f) Period-2 orbit

FIGURE 15: Circuit implementation results of four-wing chaotic attractor and period-14, period-10, period-6, period-4, and period-2 orbits.

means that the accuracy of frequency adjustment in the actual circuit can be increased $f=2.13 \times 10^{3} \omega /(2 \pi)$ times. However, comparing with the amplitude of the sine function in system (2), the amplitude of the sinusoidal signal in the circuit did not change after the variable proportion compression transformation and time scaling. So, the accuracy of amplitude adjustment was not improved. For these reasons, we select the sinusoidal signal frequency $f$ as adjusting parameters of hyperchaos.

4.2. Digital Circuit Implementation. Analog chaotic circuit and its synchronization are easily affected by precisions of the devices, interference of environment, and matching of circuit parameters. This is not conducive to engineering applications of chaos, but digital signal process technique can overcome these problems. Therefore, the digital implementation of the proposed nonautonomous system will be given in the following section based on the DSP technology.

The DSP technology is used for realizing this hyperchaotic or chaotic system, since DSP is one of the mainstream techniques for information processing at present. Moreover, the implementation of this system using DSP technology is more effective to practical applications of both chaotic 


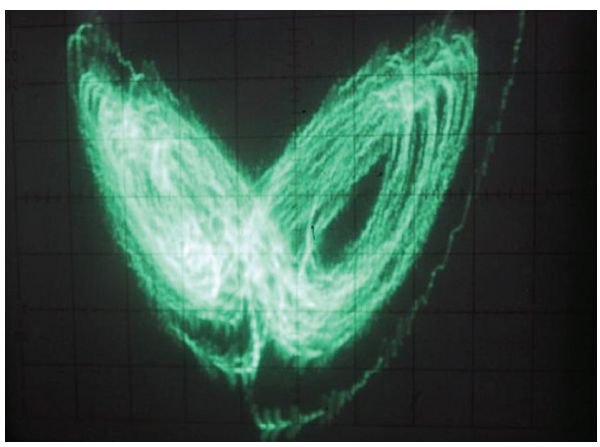

(a) Hyperchaotic attractor

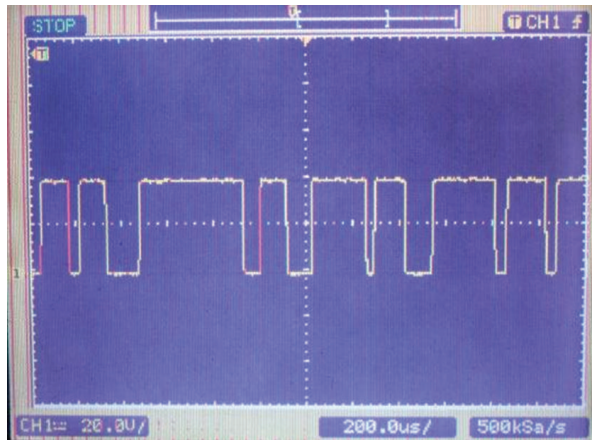

(c) Hyperchaotic binary sequence

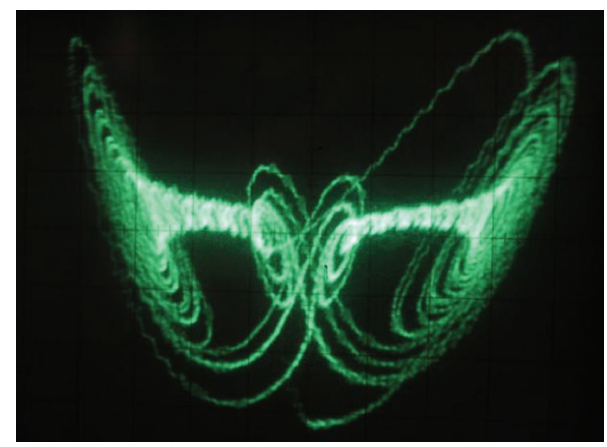

(b) Chaotic attractor

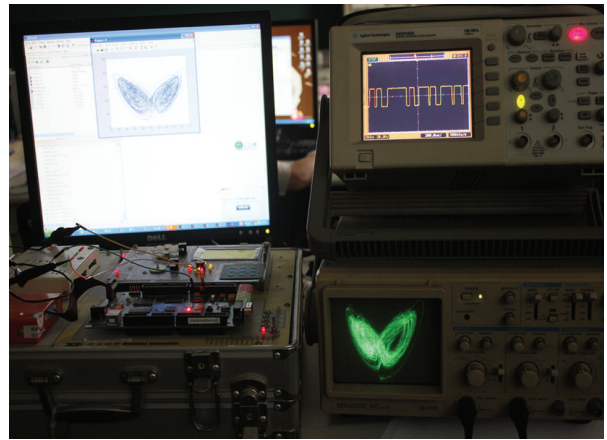

(d) Experimental facility

FIGURE 16: Experimental results and experimental facility.

PN sequence generation and chaotic information encryption/decryption processing. To this end, the iterative form of the system is given by applying Euler's method to (2):

$$
\begin{aligned}
& x_{n+1}=x_{n}-\tau A\left(x_{n}-y_{n}-a \sin \omega t_{n}\right), \\
& y_{n+1}=y_{n}+\tau\left(B x_{n}-x_{n} z_{n}-y_{n}\right), \\
& z_{n+1}=z_{n}+\tau\left(x_{n} y_{n}-D z_{n}\right) .
\end{aligned}
$$

Based on the DSP evaluation board (ICETEK-VC5509$\mathrm{AE}$ ) and the software environment platform, CCStudio_v3.3, system (2) is experimentally implemented. Figure 16 shows the experimental hyperchaotic attractor, chaotic attractor, hyperchaotic digital sequence, and the experimental facility. It is noticed that Figure 16(c) shows the hyperchaotic binary sequence generated by experimental DSP evaluation board and observed by a digital oscilloscope. The digital binary sequence can be used in various applications of information safety.

It is again noticed that rate of generated binary sequence relates to frequency of chaotic analog signals, operating frequency of digital system, quantization methods, and so on. When operating frequency of digital system and quantization method are fixed, the rate of binary sequence is also fixed. Therefore, digital implementation of chaotic system has some distinct advantages over analog circuit implementation.

\section{Conclusions}

The proposed sinusoidally driven Lorenz system can be constructed not only for generating two-wing hyperchaotic attractor, but also for four-wing chaotic attractor and high periodic orbits, through adjusting the frequency of the sine function. From the viewpoint of circuit implementation, the accuracy of frequency adjustment is usually higher than that of parameter control, and it is more convenient for circuit parameter adjustment after considering the variable proportion compression transformation and time scaling. A module-based unified circuit diagram is designed for implementing the abovementioned various hyperchaotic attractors, chaotic attractors, and periodic orbits as several typical examples for illustration and demonstration. The proposed system can generate more complex hyperchaotic signals, which can be used in the fields of various information encryptions such as secret communications and chaos cryptography as a pseudorandom signal source. The DSP implementation for the proposed system can adapt to practical applications of both chaotic pseudorandom sequence generation and chaotic information encryption/decryption processing.

\section{Conflict of Interests}

The authors declare that there is no conflict of interests regarding the publication of this paper. 


\section{Acknowledgments}

This work was supported by the National Natural Science Foundation of China under Grant 61172023, by the Specialized Research Foundation of Doctoral Subjects of Chinese Education Ministry under Grant 20114420110003, by Research Foundation of Binzhou University under Grant BZXYG1205, and by the Natural Science Foundation of Zhejiang Province under Grant LZ12F01001.

\section{References}

[1] E. N. Lorenz, "Deterministic non-periodic flows," Journal of the Atmospheric Sciences, vol. 20, pp. 130-141, 1963.

[2] O. E. Rössler, "An equation for hyperchaos," Physics Letters A, vol. 71, no. 2-3, pp. 155-157, 1979.

[3] T. Matsumoto, L. O. Chua, and K. Kobayashi, "Hyperchaos: laboratory experiment and numerical confirmation," IEEE Transactions on Circuits and Systems, vol. 33, no. 11, pp. 11431149, 1986.

[4] J. H. Lü and G. Chen, "Generating multiscroll chaotic attractors: theories, methods and applications," International Journal of Bifurcation and Chaos in Applied Sciences and Engineering, vol. 16, no. 4, pp. 775-858, 2006.

[5] C. W. Shen, S. M. Yu, J. H. Lü, and G. R. Chen, "A systematic methodology for constructing hyperchaotic systems with multiple positive Lyapunov exponents and circuit implementation," IEEE Transactions on Circuits and Systems - I, vol. 61, no. 3, pp. 854-864, 2014.

[6] X.-Y. Wang and J.-M. Song, "Synchronization of the fractional order hyperchaos Lorenz systems with activation feedback control," Communications in Nonlinear Science and Numerical Simulation, vol. 14, no. 8, pp. 3351-3357, 2009.

[7] X. Y. Wang and Y. J. He, "Projective synchronization of fractional order chaotic system based on linear separation," Physics Letters A, vol. 372, no. 4, pp. 435-441, 2008.

[8] D. Lin and X. Y. Wang, "Observer-based decentralized fuzzy neural sliding mode control for interconnected unknown chaotic systems via network structure adaptation," Fuzzy Sets and Systems, vol. 161, no. 15, pp. 2066-2080, 2010.

[9] R. Barboza, "Dynamics of a hyperchaotic Lorenz system," International Journal of Bifurcation and Chaos in Applied Sciences and Engineering, vol. 17, no. 12, pp. 4285-4294, 2007.

[10] M. J. Correia and P. C. Rech, "Hyperchaos in a new fourdimensional autonomous system," International Journal of Bifurcation and Chaos in Applied Sciences and Engineering, vol. 20, no. 10, pp. 3295-3301, 2010.

[11] L. Liu, C. X. Liu, and Y. B. Zhang, "Theoretical analysis and circuit implementation of a novel complicated hyperchaotic system,” Nonlinear Dynamics, vol. 66, no. 4, pp. 707-715, 2011.

[12] Y. X. Li, W. K. S. Tang, and G. R. Chen, "Generating hyperchaos via state feedback control," International Journal of Bifurcation and Chaos in Applied Sciences and Engineering, vol. 15, no. 10, pp. 3367-3375, 2005.

[13] X. Wang and M. Wang, "A hyperchaos generated from Lorenz system," Physica A: Statistical Mechanics and its Applications, vol. 387, no. 14, pp. 3751-3758, 2008.

[14] Y. X. Li, G. R. Chen, and W. K. S. Tang, "Controlling a unified chaotic system to hyperchaotic," IEEE Transactions on Circuits and Systems II: Express Briefs, vol. 52, no. 4, pp. 204-207, 2005.
[15] Y. X. Li, W. K. S. Tang, and G. R. Chen, "Hyperchaos evolved from the generalized Lorenz equation," International Journal of Circuit Theory and Applications, vol. 33, no. 4, pp. 235-251, 2005.

[16] K. Sun, X. Liu, C. Zhu, and J. C. Sprott, "Hyperchaos and hyperchaos control of the sinusoidally forced simplified Lorenz system," Nonlinear Dynamics, vol. 69, no. 3, pp. 1383-1391, 2012.

[17] S.-J. Cang, Z.-Q. Chen, and Z.-Z. Yuan, "Analysis and circuit implementation of a new four-dimensional non-autonomous hyper-chaotic system," Acta Physica Sinica, vol. 57, no. 3, pp. 1493-1501, 2008 (Chinese).

[18] B. Bao, P. Jiang, H. Wu, and F. Hu, "Complex transient dynamics in periodically forced memristive Chua's circuit," Nonlinear Dynamics, vol. 79, no. 4, pp. 2333-2343, 2015. 


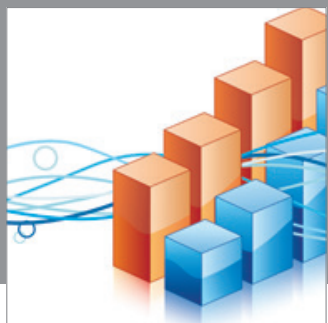

Advances in

Operations Research

mansans

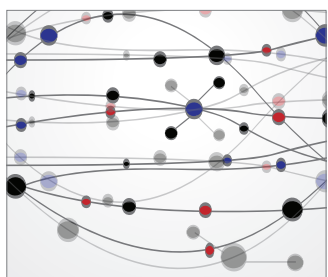

The Scientific World Journal
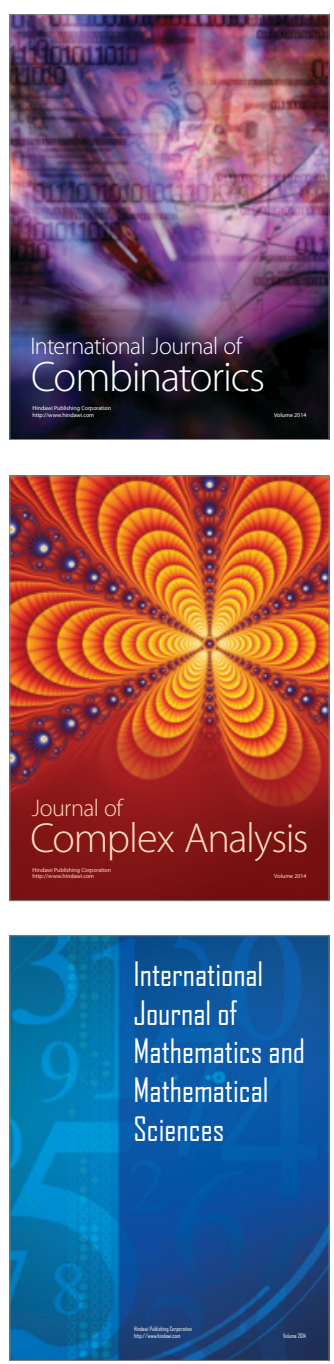
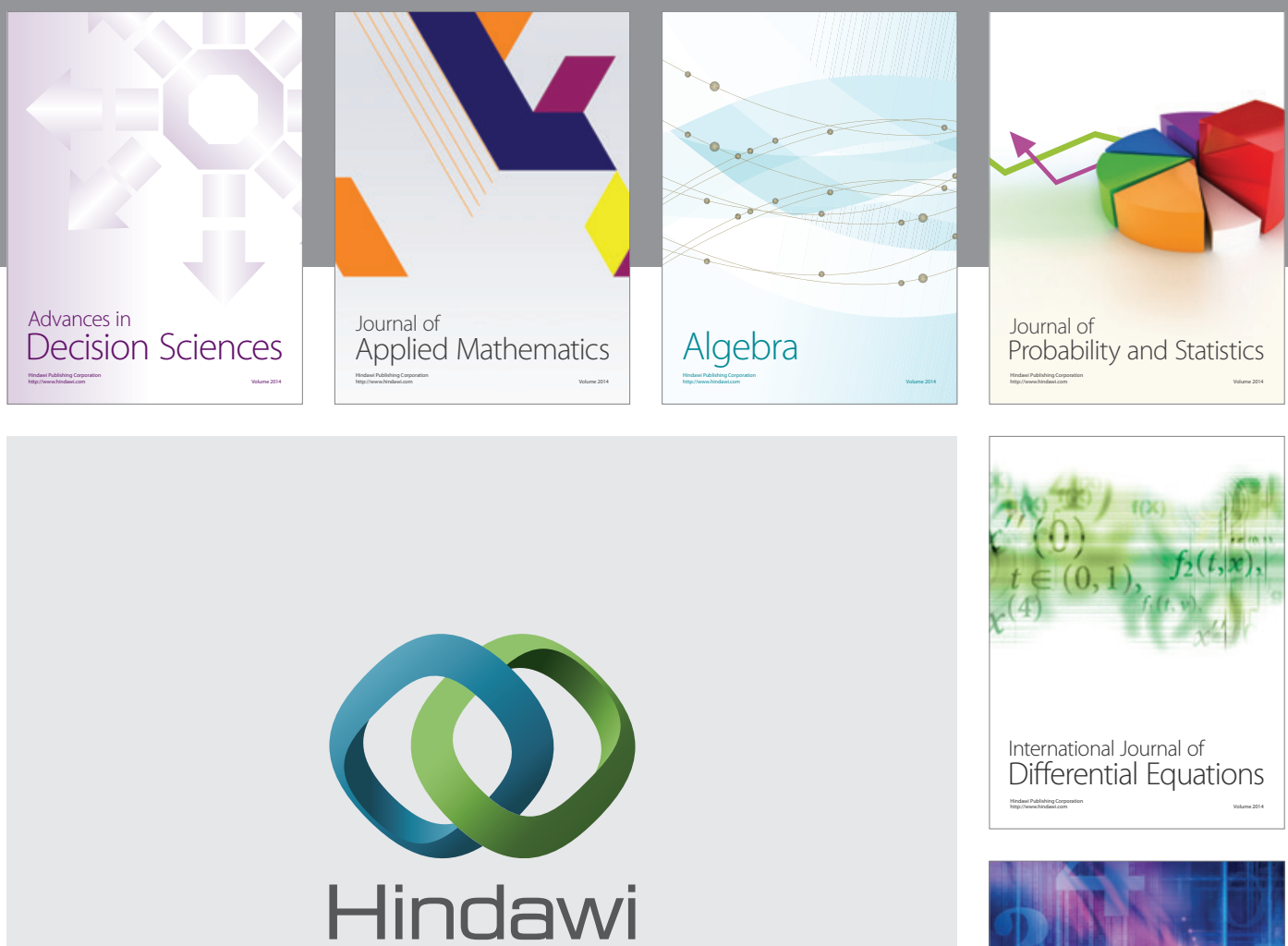

Submit your manuscripts at http://www.hindawi.com
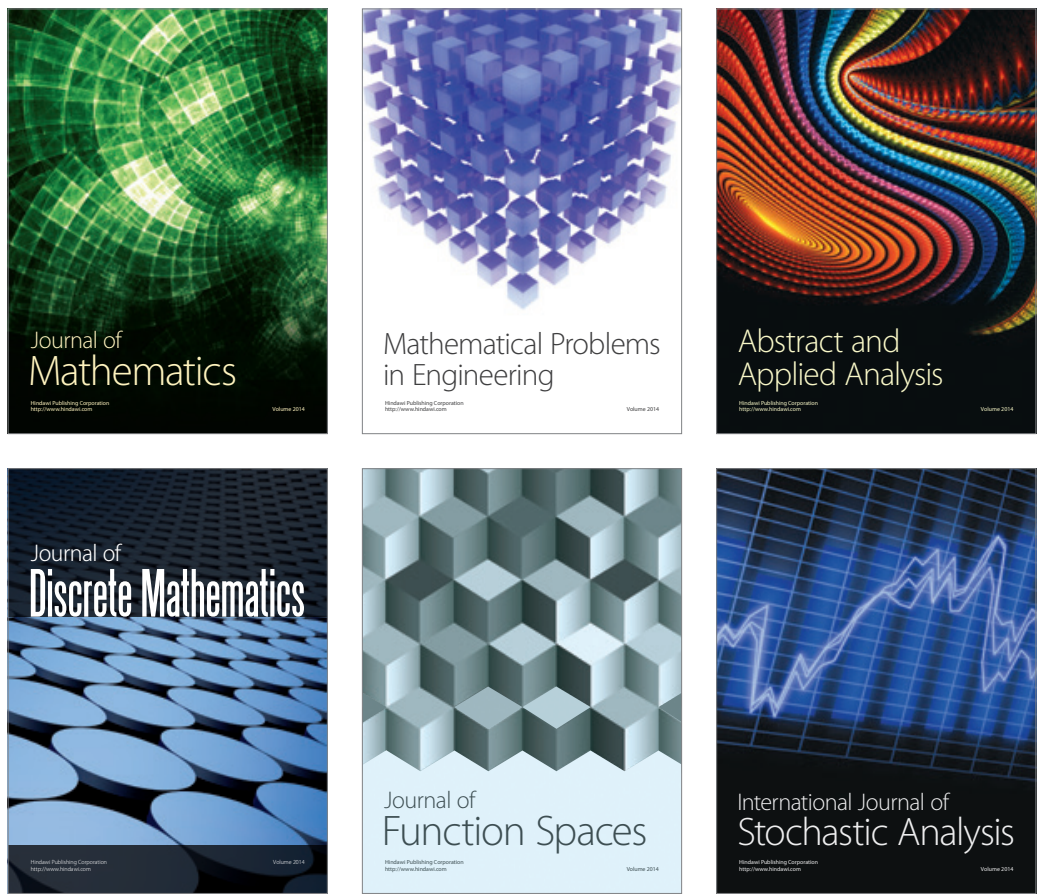

Journal of

Function Spaces

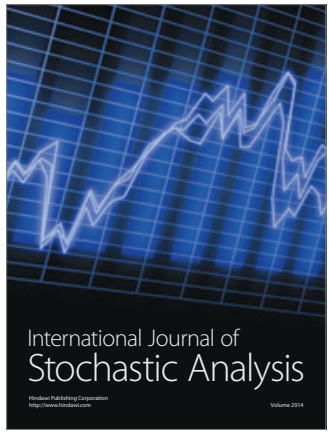

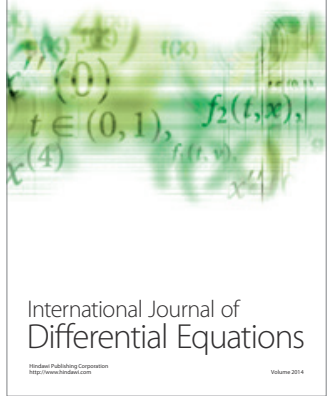
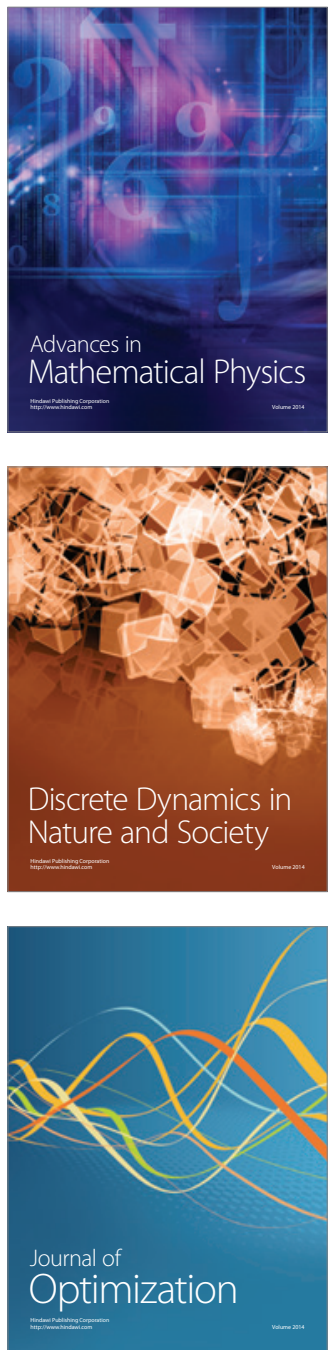